\title{
ACHETER UNE CAMÉRA INFRAROUGE
}

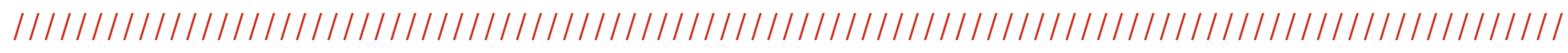

\section{Isabelle Ribet ${ }^{\mathbf{1 , 2}}$}

${ }^{1}$ ONERA-DOTA, 91761 Palaiseau cedex - France, ${ }^{2}$ IOGS, 91120 Palaiseau - France

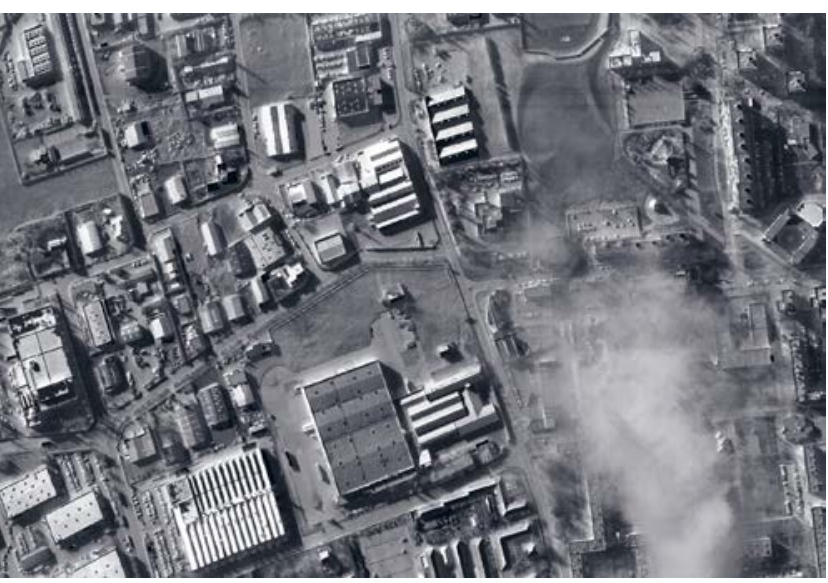

Les caméras infrarouges sont utilisées dans des domaines très variés: surveillance, déperditions thermiques, vision industrielle, domotique, voire même détection de personnes fiévreuses dans le contexte de crise sanitaire actuel... Pour couvrir des besoins aussi variés, il existe sur le marché tout un éventail de produits commerciaux, allant du petit module de vision thermique pour smartphone (poids $\sim 100 \mathrm{~g}$ et prix $\sim 200 €$ ) à la caméra refroidie très haute performance (poids de plusieurs kilos, prix allant jusqu'à plus de 100.000€).

Article publié en accès libre sous les conditions définies par la licence Creative Commons Attribution License CC-BY (https://creativecommons.org/licenses/by/4.0), qui autorise sans restrictions l'utilisation, la diffusion, et la reproduction sur quelque support que ce soit, sous réserve de citation correcte de la publication originale.

Y infrarouge est un domaine spectral très large, couvrant les longueurs d'onde comprises entre $0,8 \mu \mathrm{m}$ et $300 \mu \mathrm{m}$. Trois sousbandes spectrales, correspondant aux fenêtres de transmission atmosphérique, s'avèrent particulièrement intéressantes pour les applications mentionnées ci-dessus : la bande SWIR (pour ShortWave InfraRed, comprise entre 1 et $2.5 \mu \mathrm{m}$ ), la bande MWIR (pour MidWave InfraRed, comprise entre 3 et $5 \mu \mathrm{m}$ ) et la bande LWIR (pour LongWave InfraRed, comprise entre 8 et $12 \mu \mathrm{m}$ ).

\section{ANATOMIE}

\section{D'UNE CAMÉRA INFRAROUGE}

On retrouve dans une caméra infrarouge les mêmes éléments que dans un caméscope numérique : un objectif, une matrice de détecteurs (appelée FPA pour Focal Plane Array) et des cartes électroniques de pilotage et de remise en forme des signaux. Mais l'analogie s'arrête là. En effet,

Figure 1.

Exemples de caméras infrarouge commerciales: a) module pour smartphone; b) caméra thermique compacte portable; c) caméra thermique refroidie hautes performances. Crédit photo : (c) FLIR. lobjectif est réalisé avec des matériaux spécifiques à l'infrarouge, tels que le germanium (Ge) ou le séléniure de zinc (ZnSe). La matrice de détecteurs, quant à elle, doit utiliser un autre matériau absorbant que le silicium, qui n'est plus sensible au-delà de 1,1 $\mu \mathrm{m}$. Les semiconducteurs candidats ne manquent pas, d'où l'existence d'un nombre important de filières technologiques, chacune ayant ses avantages et ses inconvénients. a)

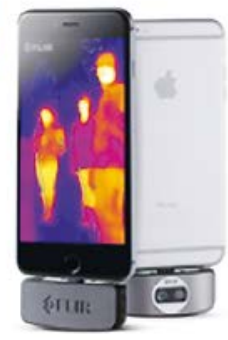

b)

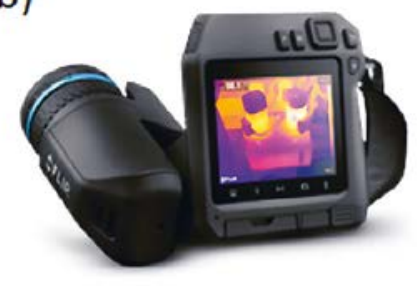

c)

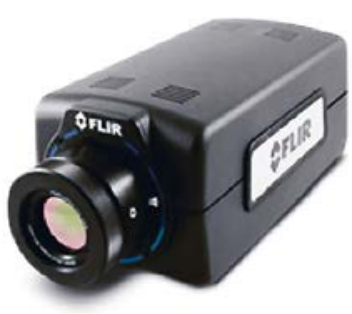




\section{PRINCIPALES FILIÈRES} DE DÉTECTEURS INFRAROUGE

On distingue deux grandes familles de détecteurs infrarouge : les détecteurs quantiques et les détecteurs thermiques.

Détecteurs quantiques : Dans les détecteurs quantiques, l'absorption d'un photon induit une transition électronique. Les alliages de semiconducteurs les plus utilisés sont : l'InGaAs

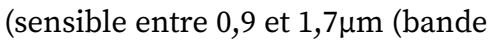
SWIR), l'InSb (sensible entre 3 et $5 \mu \mathrm{m}$ (bande MWIR)), les multipuits quantiques GaAs/AlGaAs (sensibles autour de $9 \mu \mathrm{m}$ (bande LWIR)), les super-réseaux (sensibles en bande MWIR et/ ou LWIR) ou encore l'HgCdTe (sensible, selon sa composition, en bande SWIR, MWIR et/ou LWIR). Pour obtenir l'ensemble des pixels qui constituent le circuit de détection (CD), ces matériaux peuvent être utilisés dans une structure de type photovoltaïque (photodiode) ou à barrière. Une fois le signal électrique généré, il doit être lu et remis en forme : c'est le rôle du circuit de lecture (CL), réalisé en silicium et en technologie CMOS. La connexion mécanique et électrique entre le CD et le CL, constitués de semiconducteurs différents, constitue une étape technologique complexe appelée hybridation.

Elle peut par exemple être réalisée par l'intermédiaire de microbilles d'indium (voir Fig. 2). L'une des particularités des détecteurs quantiques est que la plupart d'entre eux nécessitent d'être refroidis pour s'affranchir du courant d'obscurité (courant prenant naissance en l'absence d'éclairement incident et limitant la dynamique et le rapport signal à bruit). La température de fonctionnement requise dépend de la bande spectrale considérée : de la température ambiante à $200 \mathrm{~K}$ en bande SWIR, de $150 \mathrm{~K}$ à $80 \mathrm{~K}$ en bande MWIR, et de $80 \mathrm{~K}$ à $50 \mathrm{~K}$ en bande LWIR. Ce refroidissement est généralement obtenu grâce à une machine à froid (voire avec un simple élément Peltier pour les températures de fonctionnement les plus élevées). Cela complexifie la

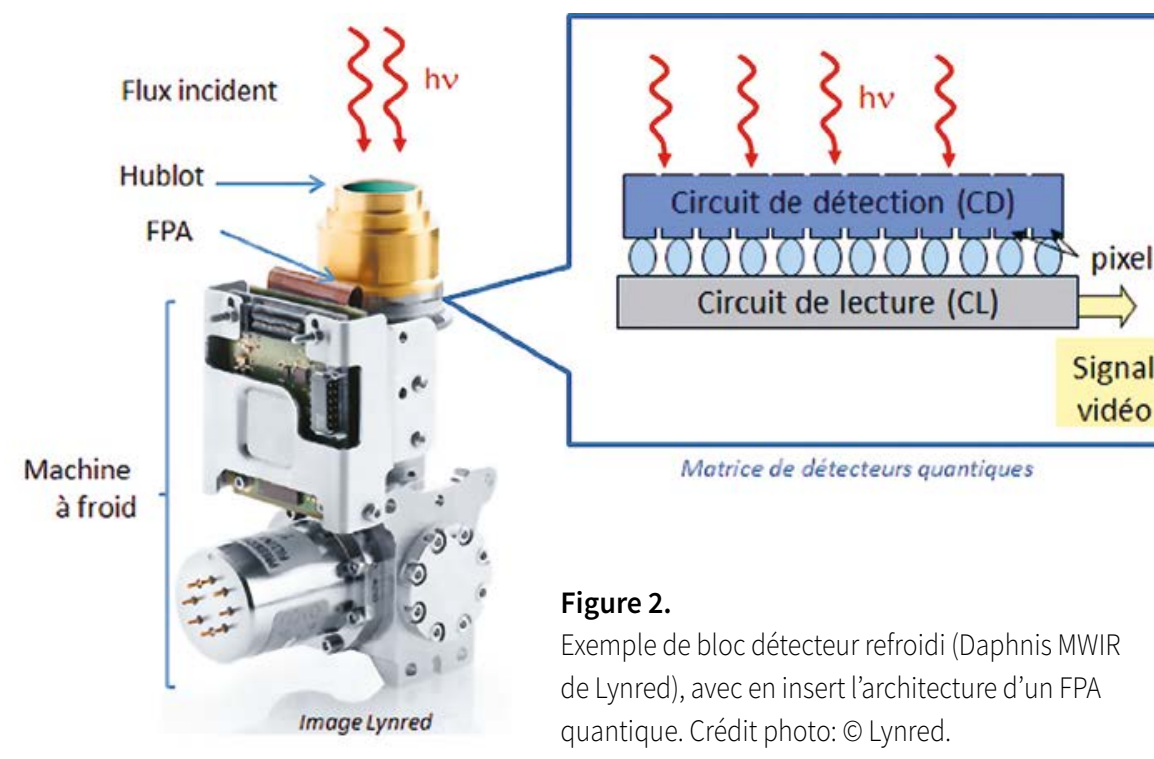

mise en œuvre de ces détecteurs, mais permet en retour d'accéder à de hautes performances.

Détecteurs thermiques : Les détecteurs thermiques constituent la deuxième grande famille de détecteurs infrarouge. Leur principe de fonctionnement est différent, puisque c'est l'échauffement induit par le flux de photons incidents sur une fine membrane qui fait varier un paramètre physique du matériau la constituant (par exemple, la résistance électrique dans le cas des microbolomètres). Contrairement à leurs concurrents quantiques, ils ne nécessitent pas de refroidissement (certains sont stabilisés en température par un élément Peltier ou TEC (pour ThermoElectric Cooler). Même si leurs performances sont en constante évolution, elles restent un peu en retrait face aux détecteurs quantiques. En revanche, les détecteurs thermiques sont plus petits, plus légers, et donc plus faciles à intégrer dans une caméra.

\section{CRITÈRES DE CHOIX D'UNE CAMÉRA INFRAROUGE}

Le premier critère à prendre en compte est le format du FPA, qui peut aller de $80 \times 60$ pixels (modules de première génération pour smartphones) à plus de $1000 \times 1000$ pixels (caméras hautes performances).
Ces formats restent bien inférieurs à ce qui se fait dans le domaine du visible où on dispose aujourd'hui de matrices de plusieurs centaines de millions de pixels. Cela est directement lié aux difficultés de réalisation technologique des détecteurs infrarouges. Le nombre de pixels requis dépend de l'application : par exemple, si on veut faire l'image d'une zone de $0.5 \mathrm{~m} \times 0.5 \mathrm{~m}$ avec une résolution millimétrique, il faudra un format supérieur à $500 \times 500$ pixels. Notons que pour une caméra donnée il est généralement possible de choisir entre plusieurs objectifs, de longueurs focales différentes. Une fois la matrice de détecteurs fixée, c'est en effet la longueur focale qui détermine le champ total vu par la caméra (souvent appelé FOV pour Field Of View, et exprimé en degrés).

Se pose ensuite la question de la bande spectrale : SWIR, MWIR ou LWIR ? Il est important de comprendre que l'origine du rayonnement n'est pas la même dans ces trois bandes: en SWIR, le signal infrarouge vient majoritairement de la réflexion de léclairage ambiant, comme dans le visible. Le contraste observé dans une image SWIR provient donc des différences de facteur de réflexion, et les images sont assez similaires aux images noir et blanc données par un appareil photo numérique, 
à part quelques inversions de contraste parfois spectaculaires (les cheveux et la végétation apparaissent blancs). La bande SWIR peut également être intéressante pour sa robustesse en conditions de visibilité dégradées (applications marines par exemple). Dans les bandes MWIR et LWIR (infrarouge dit «thermique»), le signal infrarouge vient majoritairement de l'émission propre des objets. Il est donc directement lié à leur température, ce qui permet de faire de la thermographie infrarouge. La loi du corps noir prédit que les objets à température ambiante émettent un maximum de rayonnement dans la bande LWIR, alors que la bande MWIR est, en principe, mieux adaptée à des scènes contenant des points chauds (feux de forêt, départ de missile...). Attention tout de même à la transmission atmosphérique en bande LWIR, qui sera vite dégradée en présence de vapeur d'eau. Mais surtout, ne pas oublier que le choix de la bande spectrale impactera aussi les conditions de mise en œuvre (besoin en cryogénie)... et le prix !

Les performances d'une caméra thermique sont généralement évaluées à travers sa NETD (pour Noise Equivalent Temperature Difference ou différence de température équivalente au bruit), qui est la plus petite différence de température qu'il est possible de distinguer. Les caméras thermiques atteignent des NETD de 20-40 mK, contre $15-30 \mathrm{mK}$ pour les

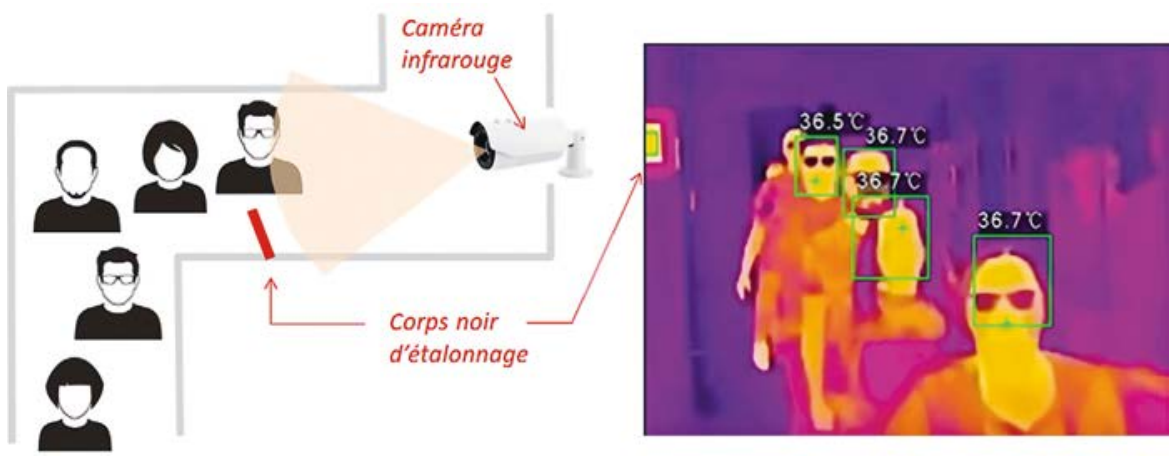

Figure 3.

Depuis 2003, des caméras thermiques sont utilisées dans les aéroports pour repérer des personnes fiévreuses.

caméras quantiques. Il est important de souligner que la NETD correspond à une mesure relative (différence de température) et ne représente pas la justesse avec laquelle il sera possible de faire une mesure de température absolue. Cette dernière est en effet plus complexe et nécessite de prendre en compte le fond instrumental. C'est la raison pour laquelle l'utilisation de caméras thermiques pour détecter les personnes fiévreuses n'est pas aussi simple qu'il y paraît, et requiert généralement l'utilisation d'un corps noir d'étalonnage dans le champ de la caméra (voir Fig. 3).

Pour les applications mettant en jeu des phénomènes rapides, la cadence image est également un paramètre de première importance.
Les cadences typiques des caméras non refroidies vont de $30 \mathrm{~Hz}$ à $60 \mathrm{~Hz}$, contre $30 \mathrm{~Hz}$ à $1 \mathrm{kHz}$ pour les modèles refroidis. Ces dernières bénéficient souvent d'un mode fenêtrage, qui peut permettre d'augmenter la cadence d'acquisition (mais en perdant en résolution).

Selon les applications, d'autres critères peuvent entrer en ligne de compte, notamment la taille, la masse et la puissance électrique consommée (SWaP en anglais, pour Size, Weight and Power). À ce niveau, l'avantage est clairement aux filières non refroidies, qui proposent les caméras portables les plus compactes. Si la caméra doit être intégrée dans un environnement sévère (essais en vol, par exemple), il faudra également porter attention à l'indice de protection (IP). Enfin, bien évidemment, reste le critère du prix : comptez de quelques centaines d'euros à $30000 €$ pour une caméra non refroidie, et de $20000 €$ à plus de $100000 €$ pour une caméra refroidie.

\begin{tabular}{|c|c|c|c|}
\hline MARQUE & PRODUITS & INFOS & DISTRIBUTEURS EN FRANCE \\
\hline Flir & $\begin{array}{l}\text { toutes caméras du SWIR au LWIR, } \\
\text { quantiques ou thermiques }\end{array}$ & info@flir.fr & $\begin{array}{l}\text { France infrarouge, Alliance Vision, Distrame, Digikey } \\
\text { Electronics, ES France, Exavision... }\end{array}$ \\
\hline Infratec & $\begin{array}{l}\text { toutes caméras du MWIR au LWIR, } \\
\text { quantiques ou thermiques }\end{array}$ & web@InfraTec.de & DB Vib Technologies \\
\hline Xenics & $\begin{array}{l}\text { toutes caméras du SWIR au LWIR, } \\
\text { quantiques ou thermiques }\end{array}$ & sales@xenics.com & Stemmer Imaging, Elvitec, Laser 2000 \\
\hline Raptor & caméras SWIR & sales@raptorphotonics.com & OptoPrim France \\
\hline Photonic Science & caméras SWIR & info@photonic-science.co.uk & Photonic Science Sales / Marketing - France \\
\hline Jenoptik & Caméras LWIR thermiques & www.jenoptik.com/contact & JENOPTIK Industrial Metrology France \\
\hline Fluke & Caméras LWIR thermiques portables & forms.fluke.com & Distrame, ES France \\
\hline EHD imaging & caméras SWIR & info@ehdimaging.de & Distrame \\
\hline Optris & caméras SWIR, LWIR & Luc.Lagorce@optris.com & $\begin{array}{l}\text { WIN Sensor, ARM, Media Mesures,Tableau mis en forme Cap } \\
\text { Instrumentation, LR Mesures, Acoris, Souplet Automatismes, } \\
\text { BFC DEXIS - Automatismes }\end{array}$ \\
\hline
\end{tabular}

